\title{
Fisiopatología de las quemaduras eléctricas: artículo de revisión
}

\author{
Physiopathology of electrical burns
}

\author{
Luis Felipe González Castro', Sandra Viviana Ávila Vargas², Jenny Tatiana Quezada Rueda³, \\ Sonia Margarita Vivas García ${ }^{4}$
}

\begin{abstract}
Electric burns are consider one of the most drastic traumas that an individual can be expose to, it compromise almost all systems of the human body. It is important to acknowledge all the general characteristics of electricity, the mechanism and the types of injuries, these are not well known by health providers. Our article is based on a bibliographic research to obtain an extend review that allows the reader comprehend the physiopathology of electric burns and apply it to the daily working basis.
\end{abstract}

\section{RESUMEN}

Las quemaduras eléctricas son consideradas uno de los traumas más drásticos al cual puede exponerse un individuo; comprometiendo directa o indirectamente a casi todos los sistemas del cuerpo humano. Es importante conocer los aspectos generales de la electricidad, los mecanismos y tipos de lesiones generadas por las mismas, que son poco comprendidos por la mayoría del personal de salud. Nuestro artículo se basa en una revisión bibliográfica para ofrecer una explicación completa, que permita al lector entender mejor la fisiopatología de este tipo de trauma y, por lo tanto, se manifieste en el actuar diario del personal de salud.

\section{Key words:}

Physiopathology,

burns,

electricity

\section{Palabras clave:}

Fisiopatología,

quemaduras,

electricidad

Médico Cirujano Plástico Reconstructivo y Estético, Hospital Universitario de Santander, Bucaramanga, Colombia.

2 Enfermera Jefe, Especialista en Atención de Enfermería del Paciente en Cuidado Crítico. Unidad de Cuidados Intensivos, Foscal Internacional, Floridablanca Santander, Colombia.

3 Médica General Servicio de Urgencias, Hospital Manuela Beltrán, Socorro, Santander.

4 Médica General de Unidad de Cuidados Posanestésicos, Hospital Universitario de Santander, Bucaramanga, Colombia.

Fecha de recepción: 24 de julio de 2018

Fecha de aceptación: 30 de agosto de 2018

\section{ORCID}

https://orcid.org/0000-0002-3745-8344

\section{Correspondencia:}

Luis Felipe González Castro:

Email: drluife@hotmail.com 


\section{Introducción}

as quemaduras eléctricas generan un tipo de lesión especial, derivadas de las propiedades físicas de la electricidad y la ruta del flujo de corriente a través de los tejidos y órganos, ocasionando compromiso superficial o compromiso multiorgánico.

A través del tiempo el ser humano siempre ha estado y estará en contacto con la corriente eléctrica debido a su uso necesario para la vida cotidiana. La mayoría de casos reportados suceden de manera accidental en el hogar o en los sitios de trabajo.

\section{Historia}

La primera muerte registrada por quemadura eléctrica fue en Lyon (Francia), cuando un carpintero en 1879 tuvo contacto con un generador de corriente alterna; así mismo años después, se conoce registro de un ciudadano norteamericano llamado Samuel W. Smith que en 1881 sufre electrocución por un generador en Buffalo, New York[1],[3].

\section{Epidemiología}

Según la OMS las quemaduras eléctricas constituyen un problema de salud pública a nivel mundial provocando alrededor de 180.000 muertes al año. En países desarrollados representan el 5-8\% del total de quemaduras[2]. Estados Unidos registra aproximadamente 1.000 muertes/año y 3.000 ingresos hospitalarios. El 4 al 6,5\% ingresan a unidad de quemados y $3-12 \%$ a salas de hospitalización general[3],[4]. El $20 \%$ de todas las lesiones ocurren en niños. La edad promedio de lesiones por electricidad 11 y 20 años; probablemente secundarios a falta de experiencia o conocimiento sobre manipulación de sistemas eléctri- cos[5]. La prevalencia es mayor en el género masculino donde ocurren $91,9 \%$ de los casos, con una relación 9:1 (masculino: femenino)[3],[6],[7]. Es la cuarta causa de muerte a nivel laboral en oficios relacionados con circuitos eléctricos o área de construcción[7] 50\% de los accidentes son de alto voltaje por contacto con líneas de alimentación y un 25\% por herramientas y máquinas en mal estado, otras causas son determinadas por ignorancia del trabajador y falta de elementos de protección laboral[3],[4],[5].

Los accidentes por corriente de bajo voltaje (60$70 \%$ ) ocurren en la población infantil 3-6 años, ocasionando lesiones en boca por mordedura de cables o por contacto con tomas de corriente en el hogar[8]. Los casos en los que se usa la electricidad con fines suicidas u homicidas, se han reportado, pero son menos frecuentes que los ya mencionados[6].

A diferencia de otro tipo de quemaduras, las eléctricas, implican para el paciente una mayor estancia hospitalaria, mayor cantidad de procedimientos quirúrgicos y gran posibilidad de muerte(7).

\section{Aspectos generales de la electricidad}

La electricidad se define como el paso de electrones de un átomo a otro y el movimiento de estos a través de un conductor es lo que se conoce como la energía eléctrica[9].

Las propiedades de la electricidad incluyen el circuito, el voltaje, la resistencia, conductancia e intensidad, los cuales influyen en los mecanismos de lesión y son predictores del estado final de la misma (Tabla 1).

1. Circuito eléctrico: Es el flujo de electrones o cargas dentro de un circuito eléctrico cerrado y se clasifica en continua-directa y alterna[3].

- Continua o directa: Es unidireccional; el flujo de electrones va hacia una misma dirección; un buen ejemplo es la generada por las baterías. Genera

\begin{tabular}{|c|c|}
\hline 1. Corriente eléctrica & $\begin{array}{l}\text { - Continua o directa } \\
\text { - Alterna }\end{array}$ \\
\hline $\begin{array}{l}\text { 2. Voltaje (Voltios) } \\
\text { Ley de Ohm } \\
V=I^{*} R\end{array}$ & $\begin{array}{l}\text { - Bajo voltaje (<1.000 voltios). } \\
\text { - Alto voltaje (>1.000 voltios) } \\
\text { - Rayo }\end{array}$ \\
\hline 3. Resistencia (Ohms) & $\begin{array}{l}\text { - Menor resistencia } \\
\text { - Resistencia intermedia } \\
\text { - Mayor resistencia }\end{array}$ \\
\hline \multicolumn{2}{|c|}{ 4. Intensidad de la corriente (Amp) } \\
\hline \multicolumn{2}{|l|}{ 5. Conductancia } \\
\hline
\end{tabular}


una sola contracción muscular que retira a la víctima de la fuente, considerándose poco peligrosa.

- Alterna: Es aquella en la que los electrones cambian de dirección de forma periódica; cada vez que estos vuelven a su posición inicial, se genera un ciclo. Ejemplo toma de corriente doméstica. Es 3 a 4 veces más peligrosa, genera estimulación de fibras musculares (40-110 veces por segundo) de forma continua ocasionando una contracción muscular sostenida llamada tetania. La corriente eléctrica entra por la mano haciendo que los músculos flexores de la misma retengan a la víctima impidiendo la liberación de su sitio de contacto, alargando el tiempo de exposición y aumentando el daño. Finalmente, esta contracción anormal, hace que los músculos torácicos imposibiliten una adecuada respiración y en el corazón se generen ritmos cardiacos mortales.

2. El voltaje: La fuerza que permite el movimiento de los electrones de un átomo a otro; cuya unidad son los voltios[9].

- Bajo voltaje (< $\mathbf{1 . 0 0 0}$ voltios): Es el tipo de accidente eléctrico más frecuente, en los niños y jóvenes. las quemaduras se presenta en manos o boca debido al contacto directo con tomas eléctricas en los hogares[10].

- Alto voltaje (> $\mathbf{1 . 0 0 0}$ voltios)[9],[11]: Lesiones térmicas por contacto con líneas de alimentación externas; pueden ser superficiales en la piel hasta lesiones profundas que comprometen músculos y órganos. Frecuente en accidentes laborales.

- Rayo[11]: Descarga eléctrica de alta tensión y amperaje pero de corta duración, asociada a alta mortalidad. La corriente masiva unidireccional se dirige al suelo atravesando el cuerpo.

Las lesiones asociadas a impacto de rayo dependen de 6 mecanismos físicos que son: golpe directo, lesión por contacto, lesión por cercanía, corriente por tierra, corriente ascendente y trauma contuso[12]. En piel se genera un signo típico, figura de Lichtenberg que se caracteriza por presentar un patrón arborescente, que desaparece a las 24 horas[11] (Figura 1).

3. La resistencia: Oposición que genera un objeto al paso de la corriente a través de sí mismo; unidad son los Ohms[10]. La resistencia de cada tejido es directamente proporcional al grado de lesión, es decir, a mayor resistencia mayor lesión[1],[9]. La piel es la primera barrera al paso de corriente. La humedad y la temperatura, determinan su resistencia[4]-[13]. Se considera un mal aislante de electricidad; si está seca es más resistente, si está húmeda reduce la resistencia generando mayor conducción de corriente eléctrica; lo que provoca mayor posibilidad de muerte por electrocución[4].

- Menor resistencia (1.500 Ohms): Músculo, nervios, vasos sanguíneos tienen agua y electrolitos buenos conductores[1].

- Resistencia intermedia: La piel seca 5.000 Ohms y la piel húmeda 1.000 ohms.

- Mayor resistencia (900.000 Ohms): Huesos, grasa y tendones tienden a calentarse y coagularse[1],[13].

4. La conductancia: Es la capacidad para transmitir la corriente[3].

5. La intensidad de corriente eléctrica: Relación entre el tiempo transcurrido y el flujo de corriente eléctrica. Unidad el amperio[9].

- 1-3 mAmp: Umbral de la percepción: corriente continua sensación de calor al contacto y en la corriente alterna hormigueo.

- 20-50 mAmp: Contracciones a nivel del musculo esquelético (Tetania). Parálisis de los músculos respiratorios.

- 50-100 mAmp: Fibrilación ventricular.

- 2,5 Amp: Asistolia.

\section{Duración de contacto}

El grado de lesión y la transformación de energía eléctrica a térmica son directamente proporcionales al tiempo en contacto con la fuente; mayor tiempo de contacto con la electricidad, mayor será la gravedad de la lesión[4].

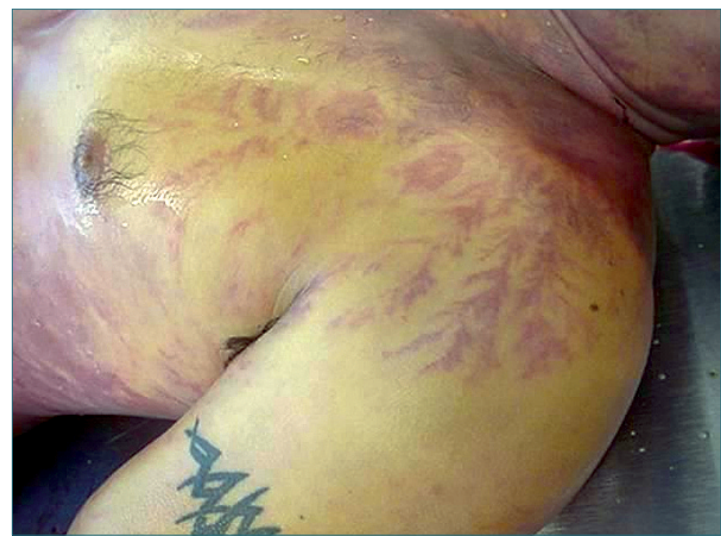

Figura 1. Signo de Lichtenberg. 


\section{Fisiopatología de la quemadura eléctrica}

Las lesiones por quemaduras eléctricas se basan principalmente en dos leyes[1]:

1. Ley de Omh: La intensidad de la corriente eléctrica es directamente proporcional al voltaje e inversamente proporcional a la resistencia del tejido afectado. La exposición de diferentes partes del cuerpo a un mismo voltaje genera una corriente diferente corriente por la resistencia que varía entre diferentes tejidos[13].

2. Ley de Joule: El calor que se genera por la corriente eléctrica es directamente proporcional a la resistencia tisular, cuanto mayor es la resistencia que ofrece un tejido al paso de la corriente, mayor es el potencial de transformación de energía eléctrica en energía térmica[14].

\section{Mecanismos de lesión}

Los 4 principales mecanismos de lesiones producto de la electricidad son[12] Tabla 2.

- Daño tisular directo: alteración en el potencial de reposo de la membrana celular generando contracción muscular (tetania).

- La transformación de energía eléctrica a térmica (ley de Joule): destrucción y necrosis coagulativa en los tejidos.

- Lesión mecánica: Traumatismos causados por violentas contracciones musculares o caídas, posterior al contacto con la corriente eléctrica.

- Teoría de la electroporación: alteración de las proteínas de las membranas celulares alterando su función e integridad[4].

Las quemaduras producidas por un agente exógeno como la electricidad, generalmente son no térmicas. La despolarización de tejidos excitables tales como el corazón causan paro cardiaco, fibrilación ventricular u otra clase de arritmias. Cuando la corriente eléctrica interactúa con los tejidos esta es transformada a térmica, generando lesión por el calor debido a la resistencia de los mismos[12]. La piel genera resistencia al flujo de corriente eléctrica produciendo calor (efecto Joule) ocasionando la carbonización de los puntos de contacto, denominados puntos de entrada y/o salida[11].

El recorrido que hace la corriente eléctrica en el cuerpo determinará las posibles lesiones; por ejemplo, una quemadura que viaja de un brazo a otro (plano horizontal) o de brazo a pierna (plano vertical), en donde el recorrido es cercano al corazón tiene mayor compromiso para la vida que una quemadura eléctrica que tenga entrada en el miembro inferior y pase a suelo[3].

Las heridas leves o superficiales que ocasiona una quemadura eléctrica pueden pasar desapercibidas, ocasionando lesiones realmente graves en órganos internos, como corazón y cerebro[12].

\section{Signo del iceberg}

Se observa una quemadura pequeña en extensión sobre la superficie corporal del primer contacto (puntiforme) y una gran lesión en tejidos profundos no evidente, signo comúnmente llamado signo del iceberg[10] (Figura 2).

\section{Tipos de lesión}

Existen 5 tipos de lesión por corriente eléctrica[3],[9],[12](Tabla 2).

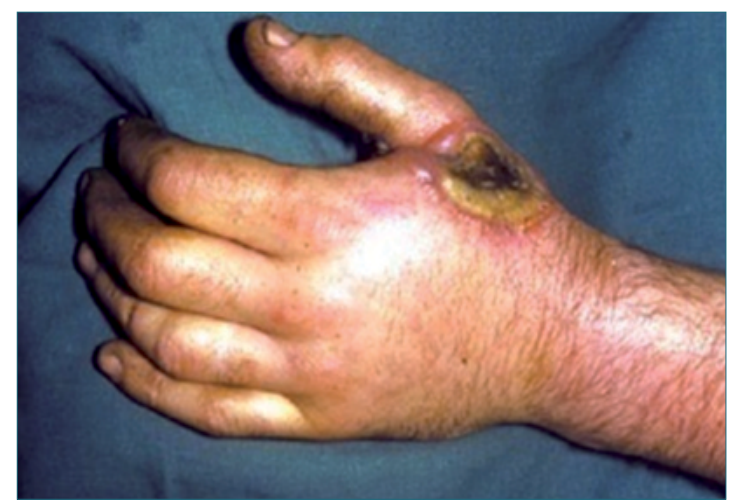

Figura 2. Signo del Iceberg.

\begin{tabular}{|c|c|}
\hline $\begin{array}{l}\text { - Daño tisular directo } \\
\text { - La transformación de energía eléctrica a térmica } \\
\text { - Lesión mecánica } \\
\text { - Teoría de la electroporación }\end{array}$ & $\begin{array}{l}\text { Tipo de lesión } \\
\text { - Lesión directa (quemadura por contacto } \\
\text { - Lesión indirecta (arco-flama y flash) } \\
\text { - Lesión electrodérmica por arco eléctrico } \\
\text { - Lesión por ignición } \\
\text { - Mixta }\end{array}$ \\
\hline
\end{tabular}


- Lesión directa (quemadura por contacto): El daño es térmico y el grado de lesión depende de la duración, frecuencia, resistencia y magnitud de la corriente eléctrica.

- Lesión indirecta (arco-flama y flash): Descargas disruptivas. La corriente eléctrica viaja por la superficie externa del cuerpo y entra en contacto con la piel húmeda o la ropa. También la corriente inicia un recorrido en un árbol, salta a un lugar de menor resistencia y, finalmente, llega a la persona.

- Lesión electrodérmica por arco eléctrico: $\mathrm{El}$ salto de la electricidad entre dos superficies que se encuentran cargadas eléctricamente y no están en contacto, genera chispa, dando como resultado un arco, cuando de los átomos del objeto conductor se genera una corriente. Frecuente en áreas donde se realiza flexión como axila, zona poplítea, muñecas y/o codos.

- Lesión por ignición: Quemadura por fuego. La corriente eléctrica incendia la ropa del individuo o algún objeto cercano y éste provoca lesión a la persona cercana o en contacto.

- Mixta: Quemadura generada por contacto directo y arco eléctrico.

\section{Compromiso por sistemas}

\section{Sistema cardiovascular}

Las quemaduras eléctricas generan dos tipos de lesiones a nivel cardiaco. Lesión directa generando necrosis en el miocardio asociada a descarga de alto voltaje; e indirecta como arritmias cardiacas [15]. La fibrilación ventricular no se revierte de forma espontánea y puede ser la causa más frecuente de mortalidad por corrientes eléctricas de bajo voltaje[4],[8],[9].

El contacto con la corriente eléctrica genera inicialmente fuga de plasma, lo que aumenta el gasto cardiaco 2 a 3 veces, con liberación de catecolaminas secundario al estrés ocasionado por la misma. Las terminaciones nerviosas cercanas a los capilares, se unen a receptores alfa adrenérgicos generando vasoconstricción de pequeños vasos sanguíneos aumentando la presión arterial[10].

Las alteraciones vasculares varían de acuerdo a su tamaño. Los grandes vasos no presentan mayor compromiso debido a que el flujo elevado permite disipar el calor generado por la quemadura eléctrica; sin embargo, puede presentar lesiones tales como necrosis, aneurismas o ruptura del mismo. Los pequeños vasos son los más afectados, en especial, los de las extremidades en donde se presenta trombosis, edema y necrosis progresiva generando limitación en el drenaje linfático con acumulo masivo de líquidos a nivel de los compartimentos corporales, provocando graves síndromes compartimentales cerrados[12].

\section{Sistema respiratorio}

El paro respiratorio es una de las causas más comunes de muerte por lesión eléctrica. La tetania de los músculos respiratorios, la inhibición del centro respiratorio en el cerebro y el paro cardiaco en pacientes con fibrilación ventricular contribuyen al paro respiratorio. Se han reportado lesiones de la vía aérea superior e inferior por inhalación de humo o gases tóxicos producto de llamas generadas por corriente eléctrica, siendo más frecuentes estos eventos en accidentes laborales[9],[12],[15].

El hemotórax, la contusión pulmonar y la lesión pulmonar aguda, secundarias a traumatismo por caída de la víctima tras el contacto con la electricidad.

\section{Sistema urinario}

La falla renal es la complicación más frecuente, resultado de depósitos en los túbulos renales de hemoglobina secundario a la destrucción de los eritrocitos y la mioglobina por destrucción tisular de musculoesquelético. Esto sumado a la isquemia de la corteza renal y disminución de la filtración por la hipovolemia generalizada, produce mayor lesión a nivel renal de difícil manejo[9],[15].

\section{Sistema musculoesquelético}

El contacto de la corriente eléctrica con el músculo, se transforma en calor generando necrosis en la zona de contacto. La lesión en la capa íntima de los vasos sanguíneos, genera como respuesta la liberación de mediadores inflamatorios como el tromboxano $A 2$, favoreciendo la vasoconstricción y formación de trombos. La falta de oxigenación conlleva finalmente a la isquemia progresiva a nivel de la microcirculación; generando necrosis[4],[9]. El 60\% de los casos con compromiso vascular y nervioso, son llevados a amputación de la extremidad[4].

En el sistema óseo se observan fracturas, dislocaciones o múltiples traumas en cráneo, tórax, abdomen, pelvis, además, de necrosis en periostio[3],[9],[12].

\section{Sistema nervioso}

Las quemaduras eléctricas pueden alterar tanto el sistema nervioso central como periférico, 80-86\%. El tejido nervioso posee una resistencia eléctrica muy baja lo que lo hace particularmente vulnerable a daño en las membranas celulares. La permeabilidad celular 
y el balance electroquímico entre los compartimentos intra y extracelular son alterados, generando desnaturalización de proteínas que lleva a edema vasogénico y daño tisular potencialmente irreversible[16].

Las descargas de alto voltaje generan pérdida del estado de conciencia, traumas craneoencefálicos y en el peor de los casos coma y/o muerte[10]. La inhibición del sistema nervioso central o hipoxia generada por alteración del sistema cardiorrespiratorio, genera isquemia cerebral y lesión medular[10].

Las alteraciones a nivel del sistema nervioso periférico son secundarias a trastornos de la conductividad eléctrica cuando hay necrosis coagulativa en el nervio (similar al músculo), o por daño indirecto en la mielina. La compresión de los vasos sanguíneos que nutren al nervio secundario al edema progresivo del síndrome compartimental, genera mayor lesión nerviosa[4].

Los síntomas iniciales son confusión, amnesia, cefalea y falta de concentración. La convulsión aparece tras el contacto y debe descartarse hipoxia o lesiones asociadas.

La lesión espinal es secundaria al traumatismo de la columna. La debilidad y las parestesias se presentan en las primeras horas del contacto. Son de buen pronóstico y recuperación.

La neuropatía periférica es frecuente en la extremidad donde paso la corriente.

\section{Sistema tegumentario}

El sitio más común de contacto primario con la corriente eléctrica es la piel de la mano y la cabeza. El punto de salida el pie.

Las quemaduras son indoloras, color amarillo-gris, deprimidas o con áreas puntiformes con necrosis a nivel central, múltiples o muy profundas en los puntos de contacto. Las quemaduras lineales suelen ser su-

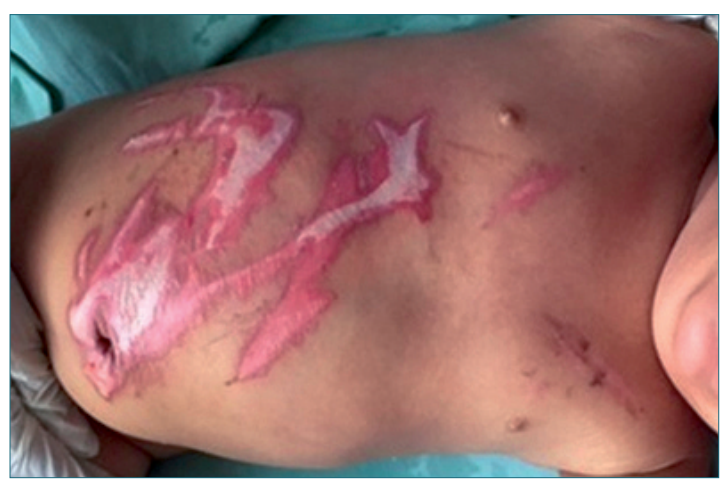

Figura 3. Quemaduras Grado II intermedias y profundas en región toraco-abdominal. perficiales y son frecuentes en lugares donde el agua y el sudor se amontonan causando vaporizaciones tales como pecho y brazo[3],[4],[15] (Figuras 3 y 4).

\section{Sistema gastrointestinal}

Lesiones por contacto directo de la electricidad con la víscera o secundario a estrés como las llamadas úlceras de curling. La vasoconstricción esplácnica ayuda a redistribuir el flujo sanguíneo hacia los órganos principales: Cerebro, corazón y pulmón, dejando hipoperfundido este sistema. Otras hemorragias intestinales, íleo paralítico, trombosis mesentérica y/o necrosis en páncreas, hígado o vesícula[9].

\section{Órganos de los sentidos}

* Oídos: Otorragia y/o perforación timpánica. La mayoría de los casos hay mejoría, o se presentan secuelas como hemotimpano, otoliquia, alteración de la cadena de huesecillo del oído y la mastoides[3],[4],[15].

* Ojos: Cataratas, 6\% de los casos de quemadura eléctrica por alto voltaje, producto de un efecto tardío causado por el realineamiento de las fibras de colágeno a nivel ocular, se presentan hasta 3 años posterior al evento, cuando la corriente eléctrica ha pasado por cabeza o cuello[9],[12].

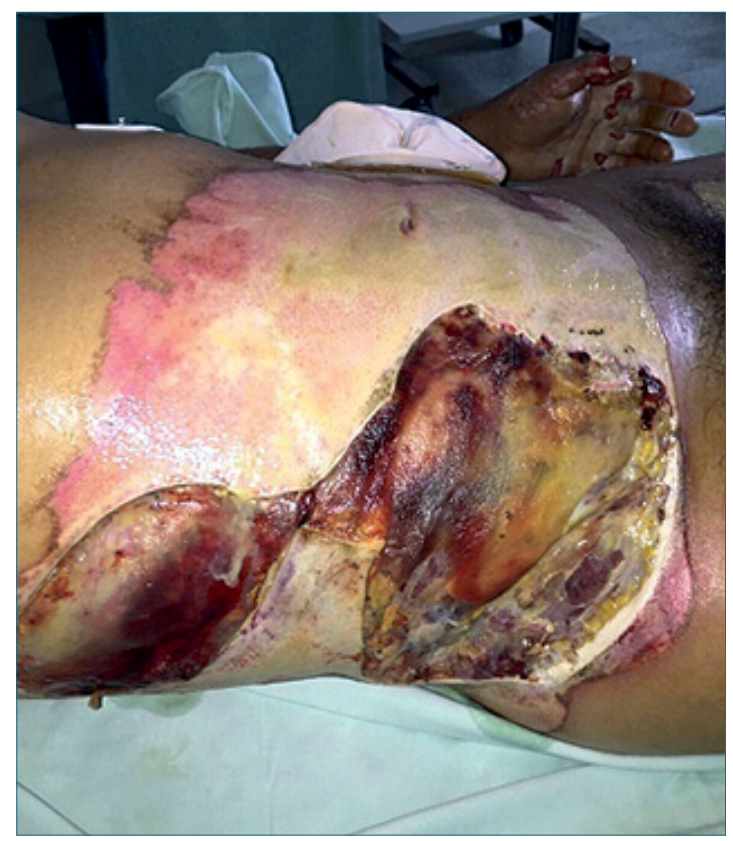

Figura 4. Quemadura Grado III en región toraco-abdominal. 


\begin{tabular}{ll} 
& Tabla 3. Lesiones por sistemas \\
\hline Cardiovascular & Arritmias cardiacas \\
Respiratorio & Paro respiratorio \\
Renal & Falla renal \\
Musculoesquelético & Síndrome compartimental \\
Nervioso & Isquemia cerebral \\
& Lesión medular \\
Tegumentario & Quemaduras grado II y grado III. \\
Gastrointestinal & Úlceras de curling \\
Órganos de los sentidos & Oído: otorragia y/o perforación timpánica \\
& Ojos: catarata \\
\hline
\end{tabular}

Otro tipo de lesiones son desprendimiento de retina, coroidoretinitis, atrofia ocular, hifema, hemorragia vítrea, iridociclitis, uveítis y lesiones corneales[4].

Choque eléctrico: Muerte súbita por fibrilación auricular y/o parálisis respiratoria por alteración del centro respiratorio[12] (Tabla 3).

Choque séptico y falla multiorgánica: Complicaciones tardías de las quemaduras eléctricas. La destrucción que se genera en los tejidos y la necrosis predispone al paciente afectado a la colonización bacteriana por lo cual se debe realizar limpieza del área, desbridamiento y/o amputación en los casos que se considere necesario para evitar complicaciones como la sepsis[15].

\section{Manejo}

Inicialmente debe trasladarse al paciente a un área segura. Desvestir y tomar medidas para prevenir hipotermia[4]. Se debe sospechar lesión medular y/o fracturas secundarias al trauma por contacto con electricidad, buscar medidas estabilizadoras hasta descartarlas. Aplicar el protocolo de soporte vital avanzado de trauma del ATLS[13].

Indagar tipo de accidente eléctrico, duración del evento y traumatismos asociados.

Valorar vía aérea, administrar oxígeno inmediato y considerar intubación orotraqueal si existen signos de falla ventilatoria o compromiso neurológico[4].

La reanimación hídrica hace parte de la estabilidad hemodinámica del paciente. La tasa de infusión inicial debe ser de $7 \mathrm{~cm}^{3} / \mathrm{kg}$ de peso $\times$ porcentaje de superficie corporal total para $24 \mathrm{~h}$; la mitad de esta estimación en las primeras $8 \mathrm{~h}$ después de la quemadura. Garantiza adecuada perfusión renal y gasto urinario de $0,5-1 \mathrm{ml} / \mathrm{kg} / \mathrm{h}$ o diuresis de $30-50 \mathrm{~cm}^{3} / \mathrm{h}[13]$. Vacunación antitetánica obligatoria.
Se recomienda la toma de electrocardiogramas seriados en busca de arritmias y signos de isquemia durante las $24 \mathrm{~h}$ del ingreso. También biomarcadores séricos aunque estos pueden estar alterados en las primeras horas del evento secundario a daño muscular.

Otros estudios función renal, electrolitos, función hepática, gases arteriales y uroanálisis.

El manejo de las heridas requiere de lavado y desbridamiento del tejido desvitalizado. Debe realizarse a las 72 h de ocurrida la quemadura. Evaluar la progresión de la necrosis tisular que puede ocasionar infección y finalmente sepsis[13].

La fasciotomía hace parte del manejo del síndrome compartimental[4].

\section{Complicaciones}

Las principales complicaciones son: Paro cardiaco, parálisis respiratoria, insuficiencia renal aguda, alteraciones neurológicas centrales y periféricas, sepsis[12]. Las complicaciones letales se dan en las primeras 24 horas, ocasionando la muerte por taquicardia ventricular, fibrilación ventricular o asistolia en un $10 \%$ de los casos[10].

\section{Conclusiones}

La fisiopatología exacta de las quemaduras eléctricas es poco entendible debido a la gran cantidad de variables que no se pueden medir, ni controlar cuando la electricidad pasa a través del cuerpo.

Así mismo, son una causa importante de morbimortalidad en la población, afectando a hombres en edad productiva; sin desconocer que cualquier individuo puede estar expuesto. Importante determinar el tiempo del impacto ya que pueden existir sistemas 
afectados de manera enmascarada. Conocer cómo responde el organismo a este tipo de quemaduras permite al médico entender mejor esta patología y lo lleva a ofrecer un manejo más completo, acertado y adecuado.

Muchas lesiones por electricidad, finalmente, es- tán asociadas a negligencia en el hogar y en el entorno laboral o por responsabilidad del trabajador.

Conflictos de interés: los autores declaran no tener conflictos de interés en la elaboración de esta revisión.

\section{Referencias}

1. Price T, Cooper MA. Electrical and Lightning Injuries. Emergency Medicine. pág.1893-1902. https://doi.org/10.1016/B978-0323-05472-0.00140-7.

2. OMS. Organización Mundial de la Salud. 2016. [actualizado 6 mar 2013; citado 24 abr 2018]. Disponible en: http://www.who. int/es/news-room/fact-sheets/ detail/burns

3. Dzhokic G, Jovchevska J, Dika A. Electrical Injuries: Etiology, Pathophysiology and Mechanism of Injury. Maced J Med Sci. 2008;1(2):54-8. https:// doi.org/10.3889/MJMS.18575773.2008.0019.

4. Rodríguez JC, Huertos MJ, RuizCabello MA, et al. Lesiones por la Electricidad y el rayo [internet]. España. Principios de Urgencias, Emergencias y Cuidados Críticos. Vol 9. Cap 9 (2)

5. Gaitan H. Quemaduras Electricas [tesis de grado] Guatemala: Universidad de San Carlos; 2017.
6. Guntheti BK, Khaja S, Uday P. Singh. Pattern of Injuries due to Electric Current. J Indian Acad Forensic Med. 2012;34(1):44488.

7. Ramos G, Ambriz A, Rodríguez $R$, et al. Resultados en el manejo de quemaduras eléctricas en un hospital de tercer nivel. Cir Gen. 2012;34(3).

8. Arnoldo B, Klein M, Gibran NS. Practice Guidelines for the Management of Electrical Injuries. Journal of Burn Care \& Research [Internet]. Oxford University Press (OUP); 2006 Jul;27(4):439-47. Available from: http://dx.doi.org/10.1097/01. bcr.0000226250.26567.4c

9. La torre W. Quemaduras eléctricas: Estudio Clínico Epidemiológico en el Hospital Nacional Guillermo Almenara Irigoyen 1997-2001 [tesis de grado]Perú: Universidad Nacional Mayor de San Marcos; 2003.

10. Luna A, Rosazza R. Paciente con Quemaduras Eléctricas, Caso Clínico. Scientifica (Cairo).
2008;6(1).

11. Tor Wo Chu, Burd A. Electrical burns. Key Topics in plastic and reconstructive surgery. Taylor \& Francis, animprint of the Taylor \& Francis Group. 2005.

12. Patiño JF. Manejo de las quemaduras eléctricas. Departamento de Cirugía, Fundación Santa Fe de Bogotá. Cap 8. Pág. 205-208.

13. Leyva JC, Carvajal F. Lesiones eléctricas, Articulo de Revision. Univ. Méd. Bogotá. 56 (1): 6374. 2015.

14. Ungureanu M. Electrocutionstreatment strategy (case presentation). J Med Life. 2014 Oct-Dec;7(4):623-6. PMID:25729443

15. Koumbourlis AC. Electrical injuries. Crit Care Med. 2002 Nov;30(11 Suppl):S424-30. https://doi. org/10.1097/00003246200211001-00007 PMID: 12528784

16. Darcia S, Solís W. Trauma Electrico. Costa Rica. Medicina legal. Vol. 33 (1).2016. 